\title{
Effectiveness of Cucumis sativus extract versus glucosamine-chondroitin in the management of moderate osteoarthritis: a randomized controlled trial
}

This article was published in the following Dove Press journal: Clinical Interventions in Aging

\section{Robert J Nash' \\ Boris KG Azantsa ${ }^{2}$ \\ Hazel Sharp' \\ Velmurugan Shanmugham ${ }^{3}$}

'PhytoQuest Limited, Plas Gogerddan, Aberystwyth, Ceredigion, UK; 2Department of Biochemistry, University of Yaoundé I, Yaoundé, Cameroon; ${ }^{3}$ Samrat Research Institute, Bangalore, Karnataka, India

Correspondence: Boris KG Azantsa Department of Biochemistry, University of Yaoundé I, PO Box 812, Yaoundé, Cameroon

Tel +237 677920184

Email borisazantsa@yahoo.fr
Purpose: Osteoarthritis (OA) is an age-related disease caused by the wear and tear of the joints. Presently, there is no known cure for OA, but its management involves the use of high doses of pain killers and antiinflammatory agents with different side and dependency effects. Alternative management strategies involve the use of high doses of glucosamine-chondroitin (GC). This study was carried out to evaluate the efficacy of Q-Actin ${ }^{\mathrm{TM}}$, an aqueous extract of Cucumis sativus (cucumber; CSE) against GC in the management of moderate knee OA.

Patients and methods: Overall, 122 patients (56 males and 66 females) aged between 40 and 75 years and diagnosed with moderate knee OA were included in this randomized doubleblind, parallel-group clinical trial that took place in three different centers. The 180 day intervention involved two groups of 61 participants in each: the GC group, which received orally the generally prescribed dose of 1,350 mg of GC twice daily and the CSE group, which received orally10 mg twice daily of CSE. The Western Ontario McMaster Universities Osteoarthritis Index (WOMAC), Visual Analog scale, and Lequesne's Functional Index were used to evaluate pain, stiffness, and physical function of knee OA in participants at baseline (Day 0) and on Days 30, 60, 90, 120, 150, and 180 .

Results: In the CSE group, the WOMAC score was decreased by $22.44 \%$ and $70.29 \%$ on Days 30 and 180, respectively, compared to a $14.80 \%$ and $32.81 \%$ decrease in the GC group. Similar trends were observed for all the other pain scores. No adverse effect was reported during the trial period.

Conclusion: The use of $10 \mathrm{mg}$ CSE, twice daily, was effective in reducing pain related to moderate knee OA and can be potentially used in the management of knee pain, stiffness, and physical functions related to $\mathrm{OA}$.

Keywords: Q-Actin ${ }^{\mathrm{TM}}$, cucumber, WOMAC, VAS, LFI, iminosugar

\section{Introduction}

Aging is a natural process accompanied by several biological and physical body alterations such as osteoarthritis (OA). ${ }^{1,2} \mathrm{OA}$ is associated with severe pain due to progressive deterioration and loss of joint lining cartilage, ligaments, tendons, and inflammation of the synovium. ${ }^{1,3}$ There is increasing evidence to suggest that OA is associated with increased pressure at the level of joints, resulting in the fragility of the cartilage matrix. This pressure is characterized by increases in cytokines and prostaglandins with consequences being the overproduction of metalloproteinases 
(MMPs) from synovium and chondrocyte bones causing lowgrade inflammation from the innate immune system. ${ }^{3-5}$

OA affects more than 100 million people and constitutes a heavy financial burden to health systems. In the United States in 2013, OA affected more than 30 million people and was the second most costly health condition managed in hospitals. ${ }^{6-8}$ The reported prevalence and incidence of OA are quite varied, but there is a consensus as to its high occurrence in adults. ${ }^{9}$ Pain in the knee joint is the most common symptom of OA. Its reported prevalence in the Indian subcontinent is $35 \%$, which is much higher in the same ethnic group living in England. ${ }^{10}$ Numerous experimental, epidemiological, and clinical studies revealed that obesity, aging, and genetic predisposition contribute to the early onset of OA. ${ }^{6,11,12}$

Various symptoms including swelling, cracking, stiffness, and pain of variable intensity are recorded in OA patients. Pain itself varies in intensity, quality, and unpredictability and is accompanied by pain's impact on mobility, mood, and sleep. ${ }^{12} \mathrm{OA}$ is sometimes accompanied by deformity, instability, and pain on patellofemoral compression. ${ }^{1,13}$ Its diagnoses is based on criteria developed by the American College of Rheumatology and by imaging techniques like the Kellgren and Lawrence scale. ${ }^{1,7,14}$

Three different scales are generally used to evaluate pain stiffness and physical function - the Western Ontario McMaster Universities Osteoarthritis Index (WOMAC), the Visual Analog scale (VAS), and the Lequesne's Functional Index (LFI). WOMAC is reliable, valid, and sensitive to the changes in the health status of patients with OA and is considered a multidimensional measure of pain, stiffness, and physical functional disability. ${ }^{15-17}$ The VAS pain scale on the other hand is a unidimensional measure of pain intensity that has been widely used in diverse populations, including those with rheumatic diseases. The VAS is simple to use and is easily adaptable to a broad range of populations and settings. ${ }^{18,19}$ Pain intensity using VAS commonly ranges from "no pain" (score 0) to "worst imaginable pain" (score of 100). ${ }^{2,20}$

The current management of OA includes the use of antidepressants, physiotherapy, and various changes in the patient's life style. Antiinflammatory injections and analgesics prescribed are often accompanied by diverse side effects. These include cases of gastrointestinal complications leading to hospitalizations and even death. ${ }^{21}$ Complementary or alternative medicine offers options to limit such adverse effects through the use of new natural and affordable products. Several randomized clinical trials (RCTs) with herbal medicines and their derivatives have been reported. These include the use of Phellodendron and
Citrus as well as Articulin-F against OA. ${ }^{11,22}$ The use of an avocado and soy bean extract known as Avocado/soybean unsaponifiable in the management of OA has also been reported. ${ }^{23,24}$ Other examples of tested natural products include capsaicin derived from hot chili peppers tested in an RCT, Devil's claw from Harpagophytum procumbens extract and ginger extracts, ${ }^{25,26}$ as well as phenolic compounds based on olive trees used in combination with moderate physical activities. ${ }^{26}$

Cucumber (Cucumis sativus L.), like melon, squash, and pumpkin, belongs to the Curbitaceae family. It is a creeping vine and a widely cultivated plant that bears cylindrical fruits used as culinary vegetables. In India, it is commonly used in traditional medicine. The presence of iminosugars has been reported in cucumber. These iminosugars can act as secondary messengers to reduce the inflammation process. ${ }^{27}$ This property could find application in the management of $\mathrm{OA}$, for which no ideal or standard drug is presently available. The present double-blind randomized study was therefore carried out to compare the efficacy of CSE, an aqueous extract of cucumber (Cucumis sativus) vs the commonly used glucosamine-chondroitin (GC) in the management of moderate knee OA.

\section{Patients and methods \\ Participants}

This study included normal weight nonhospitalized knee OA patients (males and females aged 40-75 years) willing to respect restriction to nonsteroidal antiinflammatory drugs (NSAIDS), ibuprofen, and other medications previously prescribed for their condition. They were also willing to respect all the scheduled visits and tests according to the study protocol during the study period. Patients were also expected to provide written and signed informed consent.

Excluded from the study were OA patients with history of recent clinical trial participation ( $<30$ days); patients on restricted drugs (ibuprofen, aspirin, and NSAIDS) or recreational drugs; patients with mental disorders; pregnant and lactating women; and patients with history of hypersensitivity due to allergies from particular diets (eggs and chicken) or from rescue medication (paracetamol); patients with history of GC use within the previous 3 months, as well as heavy drinkers.

Two hundred fifty-four individuals who had consulted for any form of joint pain over the previous 4 months were contacted to participate in the trials in all the centers and were assessed for eligibility. One hundred fifty applied to participate, with 122 being eligible after applying the 
inclusion criteria. Overall, 28 individuals were excluded from the study. Eighteen (eight in India, seven in Yaoundé, and three in Wales) did not meet the criteria and ten individuals (four in India and six in Yaoundé) declined to participate. Since this was a pilot study, one hundred twenty-two (122) volunteers who consented were considered as sufficient and were randomized into 61 in each group of GC and CSE, respectively.

Participants were recruited after they reviewed and understood the study details, and they then signed the IECapproved consent form. The study was in conformity with the Declaration of Helsinki (version 2013). ${ }^{28}$ The following Ethics/Institutional Review Boards approved the study: Cameroon National Ethics Committee, with Registry number 139/CNE/SE/09; PhytoQuest Ethics Committee, with reference number PhyEth/213; and the Independent Ethics Committee on Clinical Research, India, with Registry number 2016/106/IECCRI.

\section{Study design and treatments}

This study was designed as a 6-month ( 180 days) randomized, double-blind, parallel-group clinical trial. It was conducted in three centers in southern India, three centers in Cameroon, and one center in Wales. The study protocol was approved by each Institutional Ethics Board. In order to have conformities in data collection in all the centers, meetings were organized, and personnel were trained by the same research associates. Procedures of recruitment, randomization, and visits were identical in all the centers as outlined in Figure 1. Five visits were scheduled at 30-day intervals from baseline Day 1 to Day 180.

One hundred twenty-two (122) patients with symptoms of mild to moderate $\mathrm{OA}$ ( 2 and 3 on the Kellgren and Lawrence scale) selected and recruited into the study were randomly assigned to the CSE Group or GC Group. A Software CODE (IDV, Gauting, Germany) was used to generate a randomization table. Codes generated were kept confidential by the

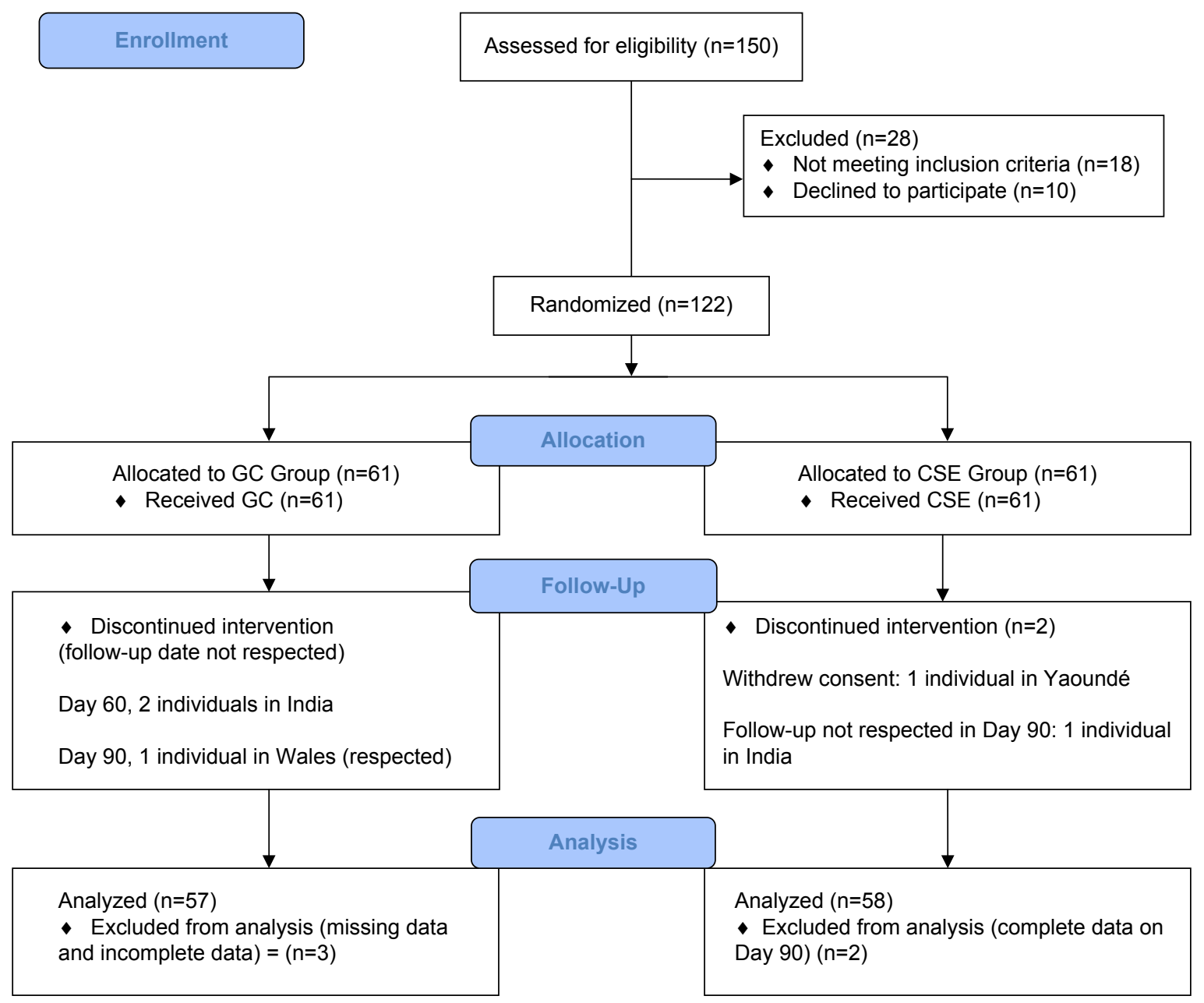

Figure I Diagram of CONSORT flow of study participants. Abbreviation: GC, glucosamine-chondroitin. 
statistician. Sixty-one patients were allocated to each group. Patients in the CSE group received $10 \mathrm{mg}$ of encapsulated CSE, twice daily, whereas those in the GC group received 1,350 mg of encapsulated GC twice daily. The CSE and GC capsules had similar appearance, shape, size, color, and odor. At the baseline, all the participants provided information on civil, demographic, nutritional status, and medical history, recorded in a questionnaire filled with the assistance of trained personnel. Follow-up evaluations were done at 30 -day intervals $(30,60,90,120$, and 180 days). The GC Group was given the dose of 2,700 $\mathrm{mg}$ per day which is the widely used dose in research on OA and provides a standard base for comparison. ${ }^{30}$

The CSE test product used in this study (sold under the tradename Q-Actin ${ }^{\mathrm{TM}}$, Lot 90115) was a proprietary aqueous extract of Cucumis sativa standardized to $\geq 1 \%$ IoBR 1 Iminosugar, and supplied by IminoTech Inc. It was manufactured under current good manufacturing practice (cGMP) conditions using a patented process that concentrates the IdoBR1 iminosugar. GC used in this study was composed of glucosamine hydrochloride (GH) and chondroitin sulfate (CS), and was purchased from Wilke Resources (Lenexa, KS, USA). The Wellable Group (Shishi City, China) manufactured GH under cGMP according to US Pharmacopeia 26 specifications. Sioux Pharm (Sioux center, IA, USA) manufactured bovine-derived CS under cGMP. CSE and GC were encapsulated in opaque size " 00 " capsules with sufficient amounts of excipients (microcrystalline cellulose and silicon dioxide) such that they were sensorially identical. Each CSE dose of $10 \mathrm{mg}$ was divided into two capsules of $5 \mathrm{mg}$ each and the remaining capsule space was filled with excipients. Each GC dose of 1,350 mg was divided into two capsules of $375 \mathrm{mg}$ of $\mathrm{GH}$ and $300 \mathrm{mg}$ of CS with the rest of space filled with excipients. The daily dose of CSE and GC was $20 \mathrm{mg}$ and 2,700 $\mathrm{mg}$, respectively. All the study materials were maintained in a secure cabinet with access restricted to the site coordinator, the dispensing pharmacist technician, and the principal investigator.

\section{Efficacy}

Efficacy measurements were assessed at all visits and included the WOMAC, VAS, and LFI indices. Subject diaries and study products were provided at all visits except on Day 180, and they were collected at all follow-up visits. Participants were instructed to record their daily consumption of the study product. Participants were required to report adverse events in their diaries, while questionnaires were administered by personnel at all study visits.
The primary endpoint was defined as the change in total WOMAC score from the baseline through Day 180 for the CSE group vs the GC group. Secondary clinical endpoints for both protocols were similar and included the change from baseline through Day 180 vs the GC group for all endpoints, including the following scores: 1) mean VAS, 2) mean WOMAC subscales, and 3) LFI. There were no changes in the trial after commencement.

WOMAC VA3.1, VAS, and LFI questionnaires were used. WOMAC questionnaire allows data collection on pain, stiffness, and physical function grouped into 05 categories for a maximum score of 100 . VAS questionnaire reports on a maximum of 70 from seven pain-related questions. LFI questionnaire allows information records on daily activities for a maximum score of $24 .^{29}$

\section{Rescue medications}

Participants were prescribed $400 \mathrm{mg}$ ibuprofen tablets (maximum $400 \mathrm{mg}$ thrice daily; total 1,200 mg) as rescue analgesia during the study based on the pain intensity reported to the study physician by certain participants. Those participants were advised not to take the rescue medicine for at least 3 days.

\section{Compliance}

Two measures of compliance were taken. Firstly, participants were asked to bring their bottles to each visit in order to record in the case report form the remaining capsules. Secondly, participants were instructed to complete a diary containing daily dosing of CSE or GC.

\section{Statistical analysis}

Results were expressed as relative score in percentage. The SAS software was used for statistical analysis of data. Efficacy of CSE was measured as reduction in pain WOMAC, VAS, LFI scores over time (from Day 0 to Day 180) and comparison with the GC group from baseline and visits. Intra-groups and inter-group comparisons were done by Wilcoxon's signed rank test to examine pair-wise changes. One-way ANOVA followed by Turkey's multiple comparison tests were conducted to study the reduction magnitude resulting from the use of CSE. Results were significant at the $95 \% \mathrm{CI}$.

\section{Results}

This study was conducted from March 2016 to September 2016. Out of 150 eligible individuals, $18.66 \%$ (28) individuals (17 males and 11 women) were excluded. One hundred 
Table I Baseline data for all patients in the study pooled from all three centers

\begin{tabular}{|c|c|c|}
\hline & GC group & $\begin{array}{l}\text { CSE }\left(Q-A c t i n^{T M}\right) \\
\text { group }\end{array}$ \\
\hline Participants in each center & 61 & 61 \\
\hline India, n (\%) & $20(32.7)$ & $23(37.7)$ \\
\hline Yaoundé, n (\%) & $25(4 I . I)$ & $26(42.62)$ \\
\hline Wales, n (\%) & $16(26.2)$ & $12(19.67)$ \\
\hline Age (years) & $52.6 \pm 5(40-73)$ & $51.9 \pm 9(42-75)$ \\
\hline \multicolumn{3}{|l|}{ Gender distribution } \\
\hline Males, n (\%) & $29(47.5)$ & $33(54.9)$ \\
\hline Females, n (\%) & $32(52.5)$ & $28(45.1)$ \\
\hline \multicolumn{3}{|l|}{ Anthropometry } \\
\hline Weight (kg) & $79.39 \pm 12$ & $82.16 \pm 11$ \\
\hline Height (cm) & $169.9 \pm 8$ & $171.1 \pm 6$ \\
\hline BMI $\left(\mathrm{kg} / \mathrm{m}^{2}\right)$ & $27.8 \pm 11$ & $28.1 \pm 10$ \\
\hline \multicolumn{3}{|l|}{ Pain intensity } \\
\hline WOMAC & $84.2 \pm 9.6$ & $83.5 \pm 8.7$ \\
\hline VAS score $(\mathrm{mm})$ & $62.5 \pm 2.8(52-71)$ & $61.0 \pm 2.2(50-69)$ \\
\hline LFI score (points) & $18.6 \pm 4.8(|5-2|)$ & $17.8 \pm 3.5(|6-2|)$ \\
\hline
\end{tabular}

Note: Results are expressed as the mean $\pm \mathrm{SD}$, percent or interquartile range. Abbreviations: BMI, body mass index; GC, glucosamine-chondroitin; LFI, Lequesne's Functional Index; VAS, visual analog scale; WOMAC, Western Ontario McMaster Universities Arthritis Index.

twenty-two $(78.70 \%)$ volunteers who consented were randomized into GC (61) and CSE (61) groups. Data collected and considered for analysis included 57 (93.94\%) and 58 (95.08\%) of participants' data in GC and CSE groups, respectively, after 180 days.

\section{Baseline data}

There was no difference $(P>0.05)$ in BMI, age, WOMAC, VAS, and LFI scores observed in the two intervention groups

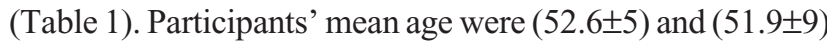
years for GC and CSE groups $(P>0.05)$, respectively. No adverse effect was reported during the study period.

\section{CSE reduced WOMAC scores more than $\mathrm{GC}$ at all time points}

Both CSE and GC improved WOMAC scores. The reduction was, however, more pronounced for the participants in the CSE group (Table 2). In the GC group, the average WOMAC score was reduced by $14.80 \%$ on Day 30 and $33.7 \%$ on Day 180 compared with the baseline value (Day 0). From baseline, CSE reduced the WOMAC score by $29.80 \%$ at Day 30 and $70.3 \%$ at Day 180. Baseline WOMAC values were not significantly different between the CSE and GC groups. These values, however, were continually reduced throughout the duration of the study in the CSE group, and were significantly $(P<0.05)$ different from the GC group at all the other time points. Changes in the GC group scores lasted only through Day 90 with no additional improvement was observed thereafter.

\section{CSE reduced VAS scores more than GC at all time points}

Both CSE and GC improved VAS scores (Table 3), with a more pronounced effect observed in the CSE group. From baseline, GC reduced the VAS by $12.34 \%$ at Day 30 and by $25.6 \%$ at Day 180 . In the CSE group, there was a $24.02 \%$ and $46.3 \%$ reduction from baseline at Day 30 and Day 180, respectively. In addition, CSE showed a continual reduction in VAS score for the entire duration of the study whereas the effectiveness of GC lasted only through Day 120 with no additional improvement thereafter.

\section{CSE reduced LFI scores more than GC at all time points}

Both GC and CSE reduced LFI scores. In the GC group, there was a $3.06 \%$ and $7.5 \%$ score reduction at Day 30 and Day 180 , respectively. In the same time periods, there was a $15.36 \%$ and $26.9 \%$ reduction in the CSE group (Table 4 ). Although there was a continual and significant $(P<0.05)$ decrease of the LFI score in the CSE group, time-related decreases from baseline values were not observed in the GC group.

\section{Discussion}

CSE, an aqueous extract of cucumber, was used in this 180 days study at a dose of $10 \mathrm{mg}$ twice daily in a doubleblind RCT involving 122 participants with moderate OA.

Table 2 Efficacy of 6 months treatment with CSE vs GC on WOMAC Score

\begin{tabular}{|c|c|c|c|c|c|c|c|}
\hline \multicolumn{7}{|c|}{ WOMAC } & \multirow{2}{*}{\begin{tabular}{|l|} 
Percentage change \\
from baseline at $D_{180}$
\end{tabular}} \\
\hline Product & $D_{0}$ & $D_{30}$ & $\mathbf{D}_{60}$ & $D_{90}$ & $\mathbf{D}_{120}$ & $D_{180}$ & \\
\hline GC & $84.2 \pm 9.6$ & $71.74 \pm 6.5$ & $66.34 \pm 7.2^{\mathrm{a}}$ & $56.58 \pm 6.9^{a}$ & $56.55 \pm 5.4^{a}$ & $55.57 \pm 8 . I^{a}$ & \begin{tabular}{|l|}
33.7 \\
\end{tabular} \\
\hline CSE & $83.5 \pm 8.7$ & $58.62 \pm 7.1^{a, b}$ & $50.02 \pm 6.6^{a, b}$ & $4 I .5 I \pm 4.8^{\mathrm{a}, \mathrm{b}}$ & $32.22 \pm 5.1^{a, b}$ & $24.8 I \pm 5.3^{\mathrm{a}, \mathrm{b}}$ & \begin{tabular}{|l|}
70.3 \\
\end{tabular} \\
\hline
\end{tabular}

Notes: Results are expressed as the mean \pm SD. ${ }^{a}$ Denotes significant difference $(P<0.05)$ between baseline and the different time points within the same group. ${ }^{b} D e n o t e s$ significant difference $(P<0.05)$ between CSE and GC for a specific time point.

Abbreviations: CSE, Cucumis sativus extract; GC, glucosamine-chondroitin; WOMAC, Western Ontario McMaster Universities Arthritis Index. 
Table 3 Efficacy of 6 months treatment with CSE vs GC on VAS score

\begin{tabular}{|c|c|c|c|c|c|c|c|}
\hline \multicolumn{7}{|l|}{ VAS } & \multirow{2}{*}{$\begin{array}{l}\% \text { change from } \\
\text { baseline at } D_{180}\end{array}$} \\
\hline Product & $D_{0}$ & $D_{30}$ & $D_{60}$ & $D_{90}$ & $D_{120}$ & $D_{180}$ & \\
\hline GC & $62.5 \pm 2.8$ & $54.7 \pm 1.6^{\mathrm{a}}$ & $50.1 \pm 2.2^{\mathrm{a}}$ & $48.7 \pm 1.8^{\mathrm{a}}$ & $39.9 \pm 2.1^{a}$ & $46.5 \pm 1.7^{a}$ & 25.6 \\
\hline CSE & $61.0 \pm 2.2$ & $46.3 \pm 1.8^{\mathrm{a}, \mathrm{b}}$ & $4 I .8 \pm 2.1^{\mathrm{a}, \mathrm{b}}$ & $38.9 \pm 1.5^{\mathrm{a}, \mathrm{b}}$ & $35.8 \pm I .4^{\mathrm{a}, \mathrm{b}}$ & $32.8 \pm I . I^{a, b}$ & 46.3 \\
\hline
\end{tabular}

Notes: Results are expressed as the mean \pm SD. a Denotes significant difference $(P<0.05)$ between baseline and the different time points within the same group. ${ }^{b} D e n o t e s$ significant difference $(P<0.05)$ between CSE and GC for a specific time point.

Abbreviations: CSE, Cucumis sativus extract; GC, glucosamine-chondroitin; VAS, visual analog scale.

No adverse effect was reported. Significantly greater reductions $(P<0.05)$ were observed in the CSE group compared with the GC group as early as from Day 30 (Tables 2-4). Previous clinical trials had demonstrated the efficacy of chondroitin and glucosamine used separately or in combination to reduce pain and stiffness and to improve physical and clinical functions in OA via overall reductions of WOMAC, VAS, and LFI scores. ${ }^{22-25}$ This study confirmed the efficacy of $\mathrm{GC}$ at a dose of $1,350 \mathrm{mg}$ twice daily in reducing the average WOMAC scores (Table 2), VAS scores (Table 3), and LFI scores (Table 4). These results are consistent with improvement in VAS scores previously reported using a combination of $1,500 \mathrm{mg}$ of glucosamine $\mathrm{HCl}, 1,200 \mathrm{mg}$ of CS, and $28 \mathrm{mg}$ of manganese ascorbate administered to 34 male military personnel with knee OA. ${ }^{26}$

The use of GC in this study also revealed a reduction in pain through a reduction of the average VAS score during the first 2 months of the study. Pain reduction in the CSE group on the other hand was sustained for the 3 months duration of the trial. This effect could be linked to the presence of bioactive compounds like the recently identified iminosugar, IdoBR1, with potential bioactive antiinflammatory activity. IdoBR1 may produce the same action as aflapin through the inhibition of 5-lipoxygenase enzyme activity, the inhibition of tumor necrosis factors- $\alpha$ (TNF- $\alpha$ ) production, or via protective action on IL-1 beta by increasing chondrocyte proliferation and increasing the synthesis of collagen and glycosaminoglycans in human primary chondrocytes. Additionally, IdoBR1 may also inhibit MMP-3 production in TNF- $\alpha$-induced human chondrocytes.

The reductions in WOMAC scores, VAS scores, and LFI scores associated with the intake of $10 \mathrm{mg}$ CSE twice daily was comparable with the results previously obtained with 30 days aflapin supplementation. ${ }^{27}$ Although the specific mechanism of action needs to be elucidated, this study represents an advancement in knowledge in the area of management of moderate OA with a low dose of aqueous cucumber extract.

\section{Limitation of the study}

Considering that the study was multicenter, data were eventually pooled due to the poor attendance in some centers. This makes interpretation of results difficult, mainly due to sociocultural and ethnic backgrounds of the participants. However, future directions include evaluations of the effects of CSE on biochemical parameters of OA like lubricin, IL-6, and MMPs levels.

\section{Conclusion}

The proprietary cucumber extract (CSE) administered at a dose of $10 \mathrm{mg}$ twice daily brought significant pain relief to participants. The low dose of this natural extract makes it a lot more attractive than conventional OA management regimes. The significant pain relief brought about by CSE was accompanied by a significantly $(P<0.05)$ improved knee pain, stiffness, and physical function over a 6-month treatment period, based on improvements in WOMAC scores, VAS scale, and LFI scores in OA patients, demonstrating its potential use and efficacy in the management of moderate knee OA.

\section{Acknowledgments}

We acknowledge the assistance and support of medical staff and personnel who assisted in data collection during follow-up, handling CRF in the different study centers in Wales, India, and Cameroon. A summary of the publication

Table 4 Efficacy of 6 months treatment with CSE vs GC on LFI score

\begin{tabular}{|l|l|l|l|l|l|l|l|}
\hline LFI & $\mathbf{D}_{120}$ & $\mathbf{D}_{180}$ & $\begin{array}{l}\% \text { change from } \\
\text { baseline at } \mathbf{D}_{180}\end{array}$ \\
\hline Product & $\mathbf{D}_{0}$ & $\mathbf{D}_{30}$ & $\mathbf{D}_{60}$ & $\mathbf{D}_{90}$ & $\mathbf{D}_{120}$ & 8.1 \\
\hline GC & $18.6 \pm 4.8$ & $18.03 \pm 6.1$ & $17.76 \pm 3.1$ & $17.49 \pm 3.6$ & $16.69 \pm 2.2$ & $17.10 \pm 2.5$ & 26.9 \\
\hline
\end{tabular}

Notes: Results are expressed as the mean \pm SD. ${ }^{a}$ Denotes significant difference $(P<0.05)$ between CSE and GC for a specific time point. ${ }^{b} D e n o t e s$ significant difference $(P<0.05)$ between baseline and the different time points within the same group.

Abbreviations: CSE, Cucumis sativus extract; GC, glucosamine-chondroitin; LFI, Lequesne's Functional Index. 
had been previously presented orally at a conference and published online at https://www.omicsonline.org/ proceedings/effectiveness-of-cucumis-sativus-extract-cseversus-glucosaminechondroitin-gc-in-the-management-ofosteoarthritis-a-rand-67692.html.

\section{Author contributions}

RJN conceived and designed the experiments, performed the experiments, and drafted the manuscript. BKGA supervised data collection in Cameroon, drafted the manuscript, and analyzed data. HS contributed to CSE composition and dosing. VS supervised data collection in India and contributed in analyzing and interpreting the patient data. All authors contributed toward data analysis, drafting and critically revising the paper and agree to be accountable for all aspects of the work.

\section{Disclosure}

The study was sponsored by IminoTech who supplied the Q-Actin ${ }^{\mathrm{TM}}$. The authors/researchers were not paid, nor did they receive any benefits from, IminoTech. Randomization and blinding minimized potential bias. The authors claim complete responsibility for the content of this publication and report no conflicts of interest in this work.

\section{References}

1. Alshami AM. Knee osteoarthritis related pain: a narrative review of diagnosis and treatment. Int J Health Sci. 2014;8(1):85-104.

2. Liu Y, Zhang H, Liang N, et al. Prevalence and associated factors of knee osteoarthritis in a rural Chinese adult population: an epidemiological survey. BMC Public Health. 2016;16:94.

3. Sokolove J, Lepus CM. Role of inflammation in the pathogenesis of osteoarthritis: latest findings and interpretations. Ther $A d v$ Musculoskelet Dis. 2013;5(2):77-94.

4. Berenbaum F. Osteoarthritis as an inflammatory disease (osteoarthritis is not osteoarthrosis!). Osteoarthritis Cartilage. 2013;21(1):16-21.

5. Saif S, Sutton P. Obesity, knee osteoarthritis and knee arthroplasty: a review. BMC Sports Sci Med Rehabil. 2013;5:25.

6. Bhatia D, Bejarano T, Novo M, Dinesh B, Tatiana B, Mario N. Current interventions in the management of knee osteoarthritis. J Pharm Bioallied Sci. 2013;5(1):30-38.

7. Kobayashi S, Pappas E, Fransen M, et al. The prevalence of patellofemoral osteoarthritis: a systematic review and meta-analysis. Osteoarthritis Cartilage. 2016;24(10):1697-1707.

8. Torio CM, Moore BJ. National Inpatient Hospital Costs: The Most Expensive Conditions by Payer, 2013: Statistical Brief \#204. Rockville, MD: Agency for Healthcare Research and Quality; 2016.

9. Allen KD, Golightly YM. Epidemiology of osteoarthritis: state of the evidence. Curr Opin Rheumatol. 2015;27(3):276-283.

10. Bliddal H, Leeds AR, Christensen R, Osteoarthritis CR. Osteoarthritis, obesity and weight loss: evidence, hypotheses and horizons - a scoping review. Obes Rev. 2014;15(7):578-586.

11. Oben J, Enonchong E, Kothari S, Chambliss W, Garrison R, Dolnick D. Phellodendron and Citrus extracts benefit joint health in osteoarthritis patients: a pilot, double-blind, placebo-controlled study. Nutr J. 2009; $8: 38$.

12. Sharma L. Osteoarthritis year in review 2015: clinical. Osteoarthritis Cartilage. 2016;24(1):36-48.
13. Smink AJ, van den Ende $\mathrm{CH}$, Vliet Vlieland TP, et al. "Beating osteoARThritis": development of a stepped care strategy to optimize utilization and timing of non-surgical treatment modalities for patients with hip or knee osteoarthritis. Clin Rheumatol. 2011;30(12): $1623-1629$.

14. Lequesne MG, Maheu E. Clinical and radiological evaluation of hip, knee and hand osteoarthritis. Aging Clin Exp Res. 2003;15(5):380-390.

15. Angst F, Aeschlimann A, Steiner W, Stucki G. Responsiveness of the WOMAC osteoarthritis index as compared with the SF-36 in patients with osteoarthritis of the legs undergoing a comprehensive rehabilitation intervention. Ann Rheum Dis. 2001;60(9):834-840.

16. Bellamy N, Buchanan WW, Goldsmith CH, Campbell J, Stitt LW. Validation study of WOMAC: a health status instrument for measuring clinically important patient relevant outcomes to antirheumatic drug therapy in patients with osteoarthritis of the hip or knee. J Rheumatol. 1988;15(12):1833-1840.

17. Gentelle-Bonnassies S, Le Claire P, Mezieres M, Ayral X, Dougados M. Comparison of the responsiveness of symptomatic outcome measures in knee osteoarthritis. Arthritis Care Res. 2000;13(5):280-285.

18. Konstantinidis GA, Aletras VH, Kanakari KA, et al. Comparative validation of the WOMAC osteoarthritis and Lequesne algofunctional indices in Greek patients with hip or knee osteoarthritis. Qual Life Res. 2014;23(2):539-548.

19. Hmamouchi I, Allali F, Tahiri L, et al. Clinically important improvement in the WOMAC and predictor factors for response to non-specific non-steroidal anti-inflammatory drugs in osteoarthritic patients: a prospective study. BMC Res Notes. 2012;5:58.

20. Mccormack HM, Horne DJ, Sheather S. Clinical applications of visual analogue scales: a critical review. Psychol Med. 1988;18(4):1007-1019.

21. Mart VL, Pergolizzi JV, Hans-Ulrich M, et al. Pain treatment in arthritisrelated pain: beyond SAIDs. Rheumatol J. 2012;6(13):320-330.

22. Kulkarni RR, Patki PS, JogVP, Gandage SG, Patwardhan B. Treatment of osteoarthritis with a herbomineral formulation: a double-blind, placebo-controlled, cross-over study. JEthnopharmacol. 1991;33(1-2) 91-95.

23. Blotman F, Maheu E, Wulwik A, Caspard H, Lopez A. Efficacy and safety of avocado/soybean unsaponifiables in the treatment of symptomatic osteoarthritis of the knee and hip. A prospective, multicenter, three-month, randomized, double-blind, placebo-controlled trial. Rev Rhum Engl Ed. 1997;64(12):825-834.

24. Maheu E, Mazières B, Valat JP, et al. Symptomatic efficacy of avocado/ soybean unsaponifiables in the treatment of osteoarthritis of the knee and hip: a prospective, randomized, double-blind, placebo-controlled, multicenter clinical trial with a six-month treatment period and a twomonth followup demonstrating a persistent effect. Arthritis Rheum. 1998;41(1):81-91.

25. Deal CL, Schnitzer TJ, Lipstein E, et al. Treatment of arthritis with topical capsaicin: a double-blind trial. Clin Ther.1991;13(3):383.

26. Szychlinska MA, Castrogiovanni P, Trovato FM, et al. Physical activity and Mediterranean diet based on olive tree phenolic compounds from two different geographical areas have protective effects on early osteoarthritis, muscle atrophy and hepatic steatosis. Eur J Nutr. Epub 2018 Feb 15

27. Bliddal H, Rosetzsky A, Schlichting P, et al. A randomized, placebocontrolled, cross-over study of ginger extracts and ibuprofen in osteoarthritis. Osteoarthritis Cartilage. 2000;8(1):9-12.

28. Nash RJ, Kato A, Yu CY, Fleet GW. Iminosugars as therapeutic agents: recent advances and promising trends. Future Med Chem. 2011; 3(12):1513-1521.

29. World Medical Association. World Medical Association Declaration of Helsinki: ethical principles for medical research involving human subjects. JAMA. 2013;310(20):2191-2194.

30. Hielm-Björkman AK, Kapatkin AS, Rita HJ. Reliability and validity of a visual analogue scale used by owners to measure chronic pain attributable to osteoarthritis in their dogs. Am J Vet Res. 2011;72(5): 601-607. 


\section{Publish your work in this journal}

Clinical Interventions in Aging is an international, peer-reviewed journal focusing on evidence-based reports on the value or lack thereof of treatments intended to prevent or delay the onset of maladaptive correlates of aging in human beings. This journal is indexed on PubMed Central, MedLine,

CAS, Scopus and the Elsevier Bibliographic databases. The manuscript management system is completely online and includes a very quick and fair peer-review system, which is all easy to use. Visit http://www.dovepress. com/testimonials.php to read real quotes from published authors.

Submit your manuscript here: http://www.dovepress.com/clinical-interventions-in-aging-journal 